\title{
Foresight in Sight
}

\author{
Santosh K. Chaturvedi $\cdot$ Krishna Prasad Muliyala $\cdot$ Devvarta Kumar
}

Received: 5 May 2019/Accepted: 6 May 2019/Published online: 10 May 2019

(C) Springer Nature India Private Limited 2019

In clinical psychiatric practice, every evaluation and assessment, makes a genuine effort to assess insight or awareness of the patients into their own illness. To a great extent, presence or lack of insight helps in differentiating between psychotic and non psychotic disorders. The mental state examination also assesses personal, social and test judgement, which also has some diagnostic value. However, clinical assessments did not attempt to evaluate a person's ability to plan future actions and there was hardly any research attention to the value of this concept, called as foresight.

Foresight is one of those cognitive capacities of human beings that distinguish us from other animals on the evolutionary ladder. The Cambridge dictionary defines foresight as "the ability to judge correctly what is going to happen in the future and plan one's actions based on this knowledge" [1]. Thus, tailoring our present behavior in order to have certain outcomes in future (e.g., investing money at the places where there is probability of higher gain later), planning as per the likely future occurrences (e.g., how to make sea water drinkable as in future there will be scarcity of water) and so on are examples of foresight. Research shows that in a limited way, some other organisms (e.g., apes and orangutans) can also manifest foresight [2];

S. K. Chaturvedi $(\bowtie) \cdot$ K. P. Muliyala · D. Kumar Psychiatric Rehabilitation Services, National Institute of Mental Health and Neurosciences, Bangalore, India e-mail: skchatur@gmail.com however, they can definitely not use foresight the way human beings do (such as complicated financial planning to have a comfortable life after retirement). The editor had a personal experience of this when a monkey snatched away his spectacles. This was a common game the monkeys played, and returned the object when offered some eatables, like biscuits. However, this time an amusing course of events was noted. When offered a biscuit, the monkey accepted it, but instead of returning the spectacles, passed it on to another monkey! The second monkey had to be offered more biscuits, only to have the spectacles passed on to a third monkey! Sensing their game plan, all other monkeys were shooed away, and a biscuit was offered to this third monkey, who then was left with no choice but to return the spectacles after breaking them. One could say their foresight made sure that other monkeys took advantage of their superior humans. In view of its significance in our life, it is imperative that the construct of foresight is researched properly. Suddendorf [2], in an article in Science writes, "It is time to carefully investigate foresight, because humans may need to get better at it if we are to continue to survive."

Among various variables that need to be researched in the context of foresight, an important variable is level of foresight in individuals diagnosed with severe mental illnesses (e.g., schizophrenia). There are a few convincing reasons for the need for such exploration. For example, patients with schizophrenia are observed 
to have problem in future planning that affects their socio-occupational functioning, the neural substrates implicated in foresight (primarily involving various prefrontal regions and cingulate cortex) are known to have impaired functionality (hypofunctional) in schizophrenia and patients' apparent apathy to significant concerns such as 'what after the caregiver's death' could be a direct result of poor foresight. Limited research shows that individuals diagnosed with schizophrenia may have impaired foresight. In a study by Eack and Keshavan [3], clinicians rated foresight of individuals diagnosed with schizophrenia or schizo-affective disorder on two items related to foresight (on a 0 to 5 points scale with higher score indicating better foresight). It was found that patients had moderate to severe foresight impairment and the impaired foresight did not have correlation with the severity of psychopathology or cognitive deficits. Also, 1 year of cognitive remediation did not have any effect on foresight. The most striking finding was that the level of foresightfulness was a significant crosssectional and longitudinal predictor of functional outcome and this was not mediated by negative symptomatology and deficits in neurocognitive function. However, a major limitation of this study is that the foresight of patients was subjectively rated by clinicians. Overcoming this limitation, in an interesting study, Lyons et al. [4] compared a group of patients with schizophrenia with healthy controls on a measure of episodic foresight that needed the participants to identify a problem, self-generate a resolution, and execute the intention at the right juncture. Findings show that patients with schizophrenia performed poorly as compared to the healthy controls. In another interesting study [5], it was found that patients with schizophrenia face difficulty in delaying gratification which could be another indicator of poor foresight as the task requires the individual to delay acquiring a desirable object to gain it in a higher quantity (immediate acquirement results in lesser quantity, e.g. one marshmallow if immediately taken and two marshmallows if one waits for a pre-decided period). To understand the neuroanatomical basis of impaired foresight in individuals diagnosed with schizophrenia Eack et al. [6] conducted a voxel-based morphometry to examine the relationship between foresight and gray matter volume in the areas implicated in foresight such as ventromedial prefrontal, orbitofrontal and cingulate cortices. They found that foresightfulness was significantly positively correlated with gray matter volume density in the right orbitofrontal, ventromedial prefrontal, and posterior cingulate cortices, as well as the left ventromedial prefrontal and anterior cingulate cortices.

Overall, these findings implicate that (a) foresight in schizophrenia is not only impaired but also is independent of severity of psychopathology and cognitive functioning (b) level of foresight can have direct bearing on the functionality of the patients (c) there should be specific intervention to improve foresight as remediation targeted to general cognitive deficits are ineffective in improving foresight in schizophrenia and (d) the neural substrate implicated in foresight may be hypofunctional in schizophrenia because of reduced volume. However, the findings can be considered preliminary as there are very few research studies in this area and, therefore, no conclusive inferences can be drawn. Further, from the rehabilitation perspective, we need to explore whether specific steps can be taken to improve the foresight of the patients and does it improve the shortand long-term psychosocial functioning of the patients.

After underscoring the need for research related to foresight in schizophrenia, we would like to highlight two major issues related to research in the domain of foresight that researchers should be aware of. First, there is a lack of consensus regarding the construct of foresight and second, there is a dearth of established tools (tested for ecological validity with established psychometric properties) for the assessment of foresight. We will briefly discuss these two issues here. So far as the lack of consensus about the construct is concerned, a number of closely related concepts such as mental time travel, episodic future thinking, prospection and delay discounting have been discussed in literature [7]. 'Prospection', 'episodic future thinking' and 'episodic foresight' involve visualization of the 'self' in future as well as require considering multiple options with decision on the optimal choice based on the previous memories. However, only the concept of 'episodic foresight' considers the importance of 'motivation' in order to attain future goals i.e. motivation to do a 'future directed action' in the present. Episodic foresight involves the ability to detach oneself from the present situation mentally and travel to the future, imagine the future scenario/goals, anticipate the needs in future, predict the most 
probable outcome (based on past memories and experiences), and travel back to present, plan for the future and execute it [8]. Episodic foresight may therefore be surmised as a higher order, metacognitive function that relies on integration of basic cognitive processes [6] and has remained a major focus of research in the domain of foresight.

So far as assessment measures are concerned, to the best of our knowledge there is lack of any norm based psychometric tool (with established validity and reliability) for assessing foresight. Most of the researchers design experiments having a component of problem that can be solved in future by taking certain steps in the present moment. Missing those steps in present moment implicate that the individual does not have foresight to tailor the present actions in a way to solve a problem in future. More specifically, the tasks involve episodic thinking i.e., thinking about a distinct temporal location and involve a 'future directed action' in the present context to achieve a future goal [9]. For example, the "spoon test" of Tulving has been used extensively to measure foresight in children [10]. Children are typically provided with a problem in one room (e.g. a locked box without a key) and then in another room there is an opportunity to select an item (e.g. key) that will solve the problem [10]. If the child fails to select the key keeping its future requirement in view, s/he won't be able to open the box when s/he goes back to the previous room. There is however, a dearth of performance-based measures of foresight in adults. Virtual week-foresight is one recently developed measure that looks at everyday situations where episodic foresight is required to problem solve [4]. These everyday situations are presented in an electronic board game. Further, to our knowledge, behavioral/performance based measures have been used in one study, to examine foresight in schizophrenia [4].

Overall, the measures of foresight in adults have broadly included content measurements and generation measurements [11]. The content measurements measure 'subject matter' and 'substance' of the simulated future scenarios, like phenomenology, specificity and episodic richness. These may be rated by the subject or an examiner. Generation measurements examine the 'elicitation of episodic future events', like the number of future events generated in a period of time, time taken to produce the episodic events.
Thus, foresight is an important aspect of cognition that needs detailed research in the context of severe mental illnesses. Factors such as effect of impaired foresight on socio-occupational adjustment of the patients, its relationship with clinical variables and development of interventions (both psychological and pharmacological) that can improve foresight are few examples of possible areas of research. Researchers are showing interest in this important domain. For example, there have been interesting developments in improving foresight in conditions such as depression by methods such as episodic specificity induction [12]. Levo-Dopa administration during episodic future thinking has shown increase in 'subjective expectation' that the future event will be more pleasurable [13]. We anticipate many such research findings to come in near future so that they aid patients on their road to recovery.

This discussion on foresight draws our attention to this issue of the Journal. The metrics from Springerlink for 2018 can be considered as more than impressive with 22,468 downloads, 41 submissions from different parts of the world and 26 accepted papers. The qualitative study from Norway reports on a thematic analysis of the role that close relationships may play in recovery processes following Substance Use Disorders. Another study from Nottingham focuses on ethnographic analysis exploring football sessions for medium-secure mental health serviceusers. The paper from Austin USA provides a synthesis on employment and education interventions targeting transition-age youth with mental health conditions. The paper from Finland investigates how two ideologies of mental health rehabilitation-recovery ideology and communal approach-are realized in interactional practices associated with psychosocial rehabilitation. Another paper from United Kingdom evaluates the impact of inpatient mental health rehabilitation on quality of life and recovery. A report from the USA describes how occupational therapy programming facilitates goal attainment in a community work rehabilitation setting. There is also an interesting user's perspective from Philadelphia USA on the beginner's crisis. Professional Culture of Mental Health Services Workers: A Meta-synthesis of Current Literature from Milan, Italy, and certification of disability from India and other countries are other papers published in this issue, further expanding its International character. 


\section{References}

1. Foresight I meaning in the Cambridge English Dictionary. https://dictionary.cambridge.org/dictionary/english/ foresight?q=Foresight. Accessed 15 Apr 2019.

2. Suddendorf T. Foresight and evolution of the human mind. Science. 2006;80(312):1006-7.

3. Eack SM, Keshavan MS. Foresight in schizophrenia: a potentially unique and relevant factor to functional disability. Psychiatr Serv. 2008;59:256-60.

4. Lyons AD, Henry JD, Rendell PG, Robinson G, Suddendorf T. Episodic foresight and schizophrenia. Br J Clin Psychol. 2016;55:107-22.

5. Heerey EA, Robinson BM, McMahon RP, Gold JM. Delay discounting in schizophrenia. Cogn Neuropsychiatry. 2007; 12:213-21.

6. Eack SM, George MM, Prasad KMR, Keshavan MS. Neuroanatomical substrates of foresight in schizophrenia. Schizophr Res. 2008;103:62-70.

7. Sujai, R. Clinical correlates of foresight in schizophrenia: a comparative study. Unpublished thesis Submited for Evaluation NIMHANS (2019).
8. Suddendorf T, Moore C. Introduction to the special issue: the development of episodic foresight. Cogn Dev. 2011;26:295-8.

9. Hudson JA, Mayhew EMY, Prabhakar J. The development of episodic foresight: emerging concepts and methods. Adv Child Dev Behav. 2011;40:95-137.

10. Scarf D, Smith C, Stuart M. A spoon full of studies helps the comparison go down: a comparative analysis of Tulving's spoon test. Front Psychol. 2014;5:893.

11. Miloyan B, McFarlane KA. The measurement of episodic foresight: a systematic review of assessment instruments. Cortex. 2018. https://doi.org/10.1016/j.cortex.2018.08.018.

12. McFarland CP, Primosch M, Maxson CM, Stewart BT. Enhancing memory and imagination improves problem solving among individuals with depression. Mem Cognit. 2017;45:932-9.

13. Miloyan B, Pachana NA, Suddendorf T. The future is here: a review of foresight systems in anxiety and depression. Cogn Emot. 2014;28:795-810.

Publisher's Note Springer Nature remains neutral with regard to jurisdictional claims in published maps and institutional affiliations. 